JOURNAL CLUB

\title{
Better survival after lung cancer surgery in high-volume hospitals
}

In this nationwide database study from England, the association between hospital volume and survival after surgery for non-small cell lung cancer (NSCLC) was investigated.

The National Cancer Data Repository was used to extract data on lung cancer diagnosed in England between 2004 and 2008. Survival status was obtained from the National Health Service Central Register and information on surgical resections was acquired from the Hospital Episode Statistics records. Socioeconomic status for each patient was estimated based on the area of residence. Comorbidity information was obtained using diagnosis codes from the Hospital Episode Statistics records and then classified according to Charlson Comorbidity Index. Hospital volume was defined as the number of patients operated on in each hospital in each year of diagnosis. Cox proportional hazards regression models were used to analyse the association between hospital volume and survival. Adjustment was made for age, sex, socioeconomic status, comorbidity and propensity to operate in the area where the patient was diagnosed.

The study included 134293 patients with NSCLC and 12862 (9.6\%) underwent lung cancer surgery at 58 hospitals. Hospital volume ranged from 1 to 194 patients per year. The proportion of NSCLC patients who received surgery varied between $5.5 \%$ and $14.3 \%$ depending on area of residence.

Higher hospital volume was associated with better survival. Specifically, this association was strongest among hospitals operating on more than 150 patients per year compared with hospitals performing less than 70 operations per year (adjusted HR 0.78 , 95\% CI 0.67 to 0.90 ). Notably, high-volume hospitals performed surgery in patients who were older and had increased comorbidity compared with low-volume hospitals. Still, early mortality was lower at these centres.

The study was limited by lack of information regarding lung cancer stage and adjuvant therapy, factors known to be associated with prognosis. The results presented in this study are consistent with prior studies reporting better survival after surgery in patients with NSCLC, and other cancers, operated on in high-volume hospitals.

- Lüchtenborg M, Riaz SP, Coupland VH, et al. High procedure volume is strongly associated with improved survival after lung cancer surgery. J Clin Oncol 2013;31:3141-6.

\section{Ulrik Sartipy}

Correspondence to Dr Ulrik Sartipy, Department of Cardiothoracic Surgery and Anesthesiology, Karolinska University Hospital, Stockholm SE-171 76, Sweden; Ulrik.Sartipy@karolinska.se

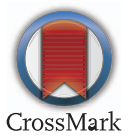

To cite Sartipy U. Thorax 2014;69:894. 